\title{
Deployment of pHealth Services upon Always Best Connected Next Generation Network
}

\author{
Georgia N. Athanasiou and Dimitrios K. Lymberopoulos \\ Wire Communications Laboratory, Electrical and Computer Engineering Department, \\ University of Patras, \\ University Campus, 26504 Rio Patras, Greece \\ gathana@ece.upatras.gr, dlympero@upatras.gr
}

\begin{abstract}
This paper deals with the deployment of personalized healthcare (pHealth) services upon new networks supporting pervasive connectivity. A profiling scheme of two levels is introduced, where health status related attributes and preferences are included in the upper-level, named Service User Profile and communication attributes compose the lower-level, named Transport User Profiles. We manage pervasive connective by means of Always Best Connected paradigm for enhancing personalization within Next Generation Network architecture.
\end{abstract}

Keywords: pHealth, NGN, ABC, AHP.

\section{$1 \quad$ Introduction}

Next Generation Healthcare paves the way for the development of ubiquitous, context aware and personalized healthcare services, known as "pHealth". The ubiquitous perspective enables healthcare to be provided at anytime and anyplace giving a sense of safety and security to the user (patient). The context-aware perspective allows the continuous assessment and regulation of any environmental and other condition that could affect the normal and productive life of the user. Finally, the personalized perspective allows the creation of healthcare services tailored to health status, living and working conditions, and several other individualized parameters of the user.

In pHealth, user is the core entity. User should not be considered as a passive receiver of healthcare service, but as a dynamic shaper in service deployment. The continuous tele-monitoring and assessment of user's health status deduces update care or treatment schemes within pHealth framework. The caregivers and all entities (subjects or objects) that are involved in these schemes should be dynamically selforganized per case. The composition of tele-working group of such entities exploiting the pervasive communication capabilities offered by advanced networks seems as the big challenge in pHealth.

This work aims to compose an overall model for user's pervasive communication in pHealth. We consider pHealth services in two operational levels. An upper level providing concrete operational modes of pHealth service that are coupled with predetermined health states of the user. User's behavior attributes, as well as preferences, needs, capabilities, occupied resources of any type, special abilities; 
social and educational status, etc compose the Service User Profile (SUP). A lower level posing user's communication requirement for any operational mode of pHealth service. The user's connectivity capabilities and attributes compose the Transport User Profile (TUP).

The proposed two levels structure is compatible with Next Generation Network (NGN) architecture, since it enables the creation of user profiles and supports qualified access into both local and wide area networks. [] We are also pursued that user's connectivity should be consistent with the Always Best Connected (ABC) paradigm for realizing personalized and ubiquitous perspective of pHealth. $\mathrm{ABC}$ oriented network is capable to provide the proper pHealth service corresponding to SUP and TUP, as well as it identifies user ubiquitously and independently of both physical location and specific equipment. [1].

\section{Personalized Services in eHealth}

The pHealth services are intended for persons (users), whose health condition is instable and therefore requires constant tele-monitoring. This instability leads person to fall from one health state to another. In each state a different treatment is required. For this reason, any pHealth service should provide concrete operational modes (service states) that should be directly tied with user's health states. The service states as well as the roles and the attributes of any person (e.g. care giver) or equipment (e.g. data base) that is involved during the execution of that state compose the pHealth SUP.

Obviously, the tele-monitoring aspects of pHealth services per service state are tightly related with the occupied networking capabilities. Service's customization generates the need for respective differentiation on service delivery. Namely, it is needed the establishment of multiple communication classes of service $(\mathrm{CoS})$, one assigned to each service state. All CoS characteristics compose the pHealth TUP that is extensively analyzed in the next sections.

In [7], we have extensively analyzed the SUP structure, while in this work we focus mainly on TUP structure. Our analysis is based on a simple pHealth service regarding the transmission of user's biosignals acquired by a BAN and occasionally medical data and examinations stored in a Body Gateway. We distinguish a service operation mode per user's health state and we established five states. In Normal State none irregular event is identified or even if it does, it is approached as insignificant for notification. In Abnormal State an abnormal but not critical event is identified. No healthcare actions are required. In Moderate State an abnormal but critical event is identified. Certain healthcare actions should be taken by user or healthcare provider. It is required transmission of user's vital signs and selected content of medical data stored in his/her Body Gateway. In Sever State, obvious and rapid deterioration is recognized in user's health status that requires service delivery of high priority. Finally, in Life-Threading State, immediate user's hospitalization is required. Communication infrastructure has to detect all the available network resources and assign to those that support service delivery of high requirements. 


\section{User Connectivity Aspects in pHealth Services}

In this paper we consider user as a communicating entity that takes profit of NGN transport capabilities, either by becoming subscriber to the proper provider or by applying nomadism on his neighboring networks.

Network subscriber: NGN is an all-IP backbone infrastructure with hierarchical wireless overlay access networks (ANs), which are structured according to the respective coverage for providing user rich accessibility [5]. User perceives the plethora of multiple existing ANs as an environment of pervasive connectivity enabling his seamless handovers. That concept paves the way to ubiquitous communications of generalized mobility, increasing simultaneously user's expectations regarding QoS.

Nomad: User acts as a communication entity surrounded by several other communication entities that can distinguish those that are actually close to, i.e. his neighbors. Neighborhood is composed by a closed group of communication entities whose features are tightly coupled with each other's concrete characteristics. Neighbors are aware of each other's capabilities and under special circumstances (i.e. emergency) can take profit of. Apparently, the above described is a degenerated approach of nomadism concept, that enables NGN user, in case of emergency, to extend the range of their pervasive connectivity by utilizing neighboring available resources.

Exploiting the above capabilities, user achieves personal mobility, i.e. user is enabled to freely communicate anytime and anywhere according to his demands [1]. In this paper, we consider that the high requirements of pHealth service that can't be satisfied by no single AN [5], they could be realized through management of pervasive connectivity, in the spirit of $\mathrm{ABC}$ paradigm. According to [6], $\mathrm{ABC}$ service performs three main signaling functions:

- AN Discovery: Any user wanting to communicate has in advance to discover the available ANs. In ABC concept, the recent research has proposed two main access discovery methods, the distributed and the centralized, as well as the combination of them [6]. In the former, user scans for the available ANs while in the latter listens for relative network announcements. There is a number of proposals regarding their implementation, but still none standardized $[2,4,6]$. Performing AN discovery, user not only discovers the available ANs but also acknowledges the supported QoS.

- $\quad$ Personalized Selection of Best AN: User evaluates the discovered ANs and he selects the best one (or ones) for his communication. Because of the complexity of this problem, Multi-Constraint Decision Method (MCDM) and Multi-Attribute Decision Method (MADM) are deployed. [2] It is a set of methods tailored to decision problems highly dependent on the presence of a number of decision criteria.

- $\quad$ Seamless horizontal and vertical handover: User performing IEEE 802.21 can QoS guaranteed seamless handover within the same AN (horizontal) and among different ANs (vertical). 
Considering NGN as the underlying networking environment of pHealth services, we propose that ABC service should be incorporated into the Transport Stratum of NGN and especially into the Network Attachment Control Function (NACF) that is standardized in ITU-T Y.2014 NACF manages and controls NGN transport resources assigned to user through ANs. User acquires ABC service through a framework which gives him the possibility of always exploiting the requested service through the best access technology and the best terminal available [4].

\section{The Proposed Structure of Transport User Profile (TUP)}

In this paper we focus on $\mathrm{ABC}$ service function of $\mathrm{AN}$ Personalized Selection for integrating pHealth service. Function's effective performance requires the pHealth to provide that information confining personalization, i.e. the user's exigencies, functions of his profile and personal preferences, all oriented in SUP [3].

According to ITU-T Y.2014, NACF establishes TUP, which includes all communication capabilities and preferences of the user. User's behavior is demonstrated via TUP's content, which is mapped into the selection criteria that $\mathrm{ABC}$ service utilizes for choosing the best AN. Selection Criteria are the Decision Factors on best AN decision making. We consider this mapping process as the cornerstone upon which pHealth services should be established.

\subsection{TUP Functionality}

ITU-T Q Supplement 58 defines that TUP includes general rules and QoS parameters both determining network attachment and resource allocation. We adjust TUP's content to meet pHealth's requirements for decisively enhance it. According to SUP, each user joins pHealth services playing a specific role that is translated in communication terms into establishment of multimedia sessions of variable QoS. For example, the session for transferring patient's vital signs is differentiating on QoS requirements from the emergency session for transmitting treatment plans. In this paper, we associate each such service state with a connectivity scheme. The content of TUP is differentiated per user role and service state, generating connectivity schemes. The set of different connectivity schemes is mainly the content of TUP. In this essay we construct the internal structure of TUP on the basis of the above described ABC paradigm. Hence, we encapsulate intelligence in TUP through the following Rules and Decision Factors:

Rule 1: It is a rule for determining whenever user is permitted to access neighboring ANs besides the subscribed ones. Notice that the ABC AN Discovery function renders user aware about the whole set of surrounding ANs (subscribed and neighboring). This rule applying e.g. EXCLUDE / INCLUDE algorithm, delimits the plethora of discovered ANs to the actual available to user The importance of that rules lies on the fact that it paves the way for ubiquitous communications, while restricting user to abuse neighboring ANs. Only in emergency situations, user is permitted to access them. 


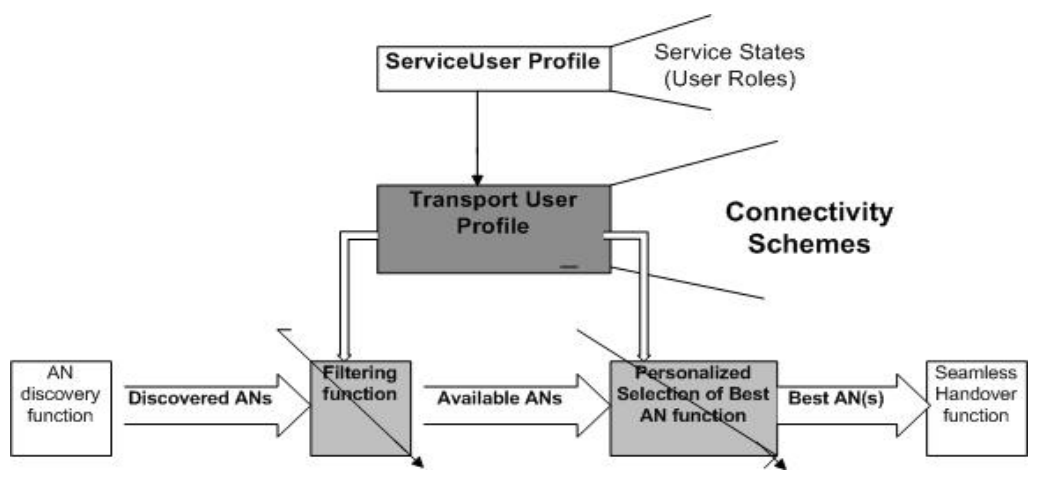

Fig. 1. ABC service

- $\quad$ Rule 2: A rule defining the connectivity mode, i.e single-homed or multi-homed, user can apply above the available ANs. Multi-homed is the user that simultaneously establishes and sustains multiple connections (using multiple IP addresses), one per available ANs.

- Decision Factors: Depending on the application are defined the following six (6) Decision Factors affecting final decision for best AN among the alternative discovered ANs; Availability (containing RSS and Coverage Area), Throughput, Timeless (containing Delay, Respond Time and Jitter), Security, Cost and Reliability (containing BER, burst error, Average number of retransmission per packet and Packet Loss Ratio). Note that each Decision Factor has not the same importance for every class of service $(\mathrm{CoS})$ and that should be declared through a ranking process. That means, within each connectivity scheme each factor has a different influence in $\mathrm{ABC}$ process (weight factor).

\subsection{TUP for Personalizing ABC Service}

Fig. 1 depicts the proposed structure of ABC service incorporating TUP. By means of this structure the personalized user's connectivity is realized through discrete steps. In first place, ABC AN Discovery function detects all the subscribed and neighboring provided ANs. In this point, we introduce a Filtering function, for extracting the available ANs from the discovered ones. The Filtering function applies the Rule-1 defined in TUP. As the subscribed ANs are considered a priori available, Rule-1 is applied on neighboring ANs. The output of Filtering function is the input to $A B C$ Personalized Selection of Best AN function. For the implementation of this function we apply the Analytic Hierarchy Process (AHP), a method to analyze complex problems, through structuring hierarchies of multiple criteria. [4] Applying AHP, we formulate the internal structure of this function as an hierarchy, where at the top are placed the best ANs (output), as being the overall objective and at the bottom are located the available ANs (input), as being the Decision Alternative In the middle of hierarchy are the six (6) Decision Factors. These factors are synthesized through a four (4) steps method for indicating the resulting rank of best ANs. [2] In step 1, the Decision Alternatives (available ANs) are scored under each Decision Factor. It is a 
pair-wise judgment, ${ }^{\alpha_{i, j}}$ of importance of $\mathrm{i}$ Decision Factor over $\mathrm{j}$ Decision Factor. The judgment takes value from the fundamental five-point scale, posed by AHP in [4] and shown in text box below. The pair-wise comparison is represented through a judge-matrix Aixj. Note that:

$$
\alpha_{j, i}=1 / \alpha_{i, j} \text { and } \alpha_{i, i}=\alpha_{j, j}=1 \text {. }
$$

Table 1. Scale of preference levels

\begin{tabular}{ll}
\hline Performance Level & Numerical Value \\
\hline Equally & 1 \\
Moderately & 3 \\
Strongly & 5 \\
Very Strongly & 7 \\
Extremely & 9 \\
\hline
\end{tabular}

TUP set partially formulates the judge matrix by setting the Decision Criteria in the appropriate row and column, depending on their influence to the final decision:

$$
\left[\begin{array}{ccc}
\alpha_{1,1} & \ldots & \alpha_{1, j} \\
\vdots & \ddots & \vdots \\
\alpha_{i, 1} & \ldots & \alpha_{i, j}
\end{array}\right]=A_{i \times j}
$$

In step 2, the weights of Decision Factors are determined, i.e. it is composed the weight vector $\mathrm{W}=[\mathrm{w} 1, \mathrm{w} 2, \ldots, \mathrm{wn}]$, where " $\mathrm{n}$ " is the number of Decision Factors. Vector $\mathrm{W}$ is recovered from matrix $\mathrm{A}$ by finding the solution of $\mathrm{AW}^{\mathrm{T}}=\mathrm{W}^{\mathrm{T}} \mathrm{u}$, or by applying the following method: Normalizing each column $\mathrm{j}$ of matrix A:

$$
\alpha_{i, j}^{\prime}=\alpha_{i, j} / \sum_{i=1}^{n} \alpha_{i, j}^{\prime}
$$

Creating a vector with element the sum of each row i of matrix A:

$$
\alpha_{i}^{\prime \prime}=\sum_{j=1}^{n} \alpha_{i, j}^{\prime}
$$

Finally the weight of Decision Factor "i" in the weight vector $\mathrm{W}$ is:

$$
\mathrm{w}_{\mathrm{i}}=\alpha_{\mathrm{i}}^{\prime \prime} / \sum_{\mathrm{ji}=1}^{\mathrm{n}} \alpha_{\mathrm{j}}
$$

In step 3, the consistent of matrix A should be tested. In step 4, the final score, i.e. the final rank of Decision Alternatives is produced by synthesizing the priorities (Decision Factors). The decision-making vector is $\mathrm{R}=\mathrm{WxA}$, where the element of the greater value is the most appropriate for choice. The number of selected best ANs from the $\mathrm{R}$ vector is defined through Rule-2. In single-homed case, it is selected only 
the best $\mathrm{AN}$ (the element of the greatest value in $\mathrm{R}$ vector), while in the multihomed it is selected a subset of the top ANs.

The necessity for a network profiling system is inherently tided with the nature of personalized services. Therefore all networks supporting personalized services should incorporate a profiling system. In this essay, we utilize the primitive profiling system NGN provided, however we are pursued that any communication system handling the network access capabilities should incorporate it.

\section{Connectivity Schemes Supporting pHealth}

The above analyzed TUP structure provides pHealth services the capability to determine how network resources should be handled for achieving the required QoS. As argued above, TUP is differentiated per service state and user role, generating connectivity schemes. In this point we consider that pHealth services should be mapped into four (4) different connectivity schemes; Non-emergency, Notification, Alert and Alarm. We concluded to this number and schemes type as a first approach and could be modified in the future.

Table 2. Connectivity schemes for the examined pHealth service

\begin{tabular}{|c|c|c|c|}
\hline $\begin{array}{l}\text { Connectivity } \\
\text { Schemes }\end{array}$ & Rule-1 & Rule-2 & $\begin{array}{l}\text { Ranking of Decision } \\
\text { Factors }\end{array}$ \\
\hline Non - Emergency & $\begin{array}{l}\text { EXCLUDE } \\
\{\text { NEIGHBORING AN }\}\end{array}$ & MISO & $\begin{array}{c}\text { Availability }=\text { Cost }> \\
\text { Reliability }>\text { Security }> \\
\text { Throughput }>\text { Timeless }\end{array}$ \\
\hline Notification & $\begin{array}{l}\text { EXCLUDE } \\
\{\text { NEIGHBORING AN }\}\end{array}$ & MIMO & $\begin{array}{c}\text { Availability }=\text { Cost }> \\
\text { Throughput=Reliability }> \\
\text { Timeless }>\text { Security }\end{array}$ \\
\hline Alert & EXCLUDE $\{$ NONE $\}$ & MIMO & $\begin{array}{c}\text { Timeless }>\text { Availability }= \\
\text { Throughput }>\text { Reliability }> \\
\text { Security }>\text { Cost }\end{array}$ \\
\hline Alarm & EXCLUDE $\{$ NONE $\}$ & MIMO & $\begin{array}{c}\text { Timeless }=\text { Throughput }> \\
\text { Availability }>\text { Reliability }> \\
\text { Security }>\text { Cost }\end{array}$ \\
\hline
\end{tabular}

In this section we depict how TUP rules are applied in each scheme. We consider, as an example, the pHealth service described in section II that is deployed into five (5) service states. The selection of the appropriate AN or ANs that satisfy the communication needs of each state, is the main target. According to the proposed profiling concept, first we map each one of these states into a particular connectivity scheme. The states "Normal" and "Abnormal" are handled by the "Non-emergency", while "Moderate", "Severe" and "Life Threatening" states are handled by "Notification", "Alert" and "Alarm" schemes, respectively. In Table 1 we depict how Rule-1, Rule-2 and Decision Factors are formulated in each scheme of the current example. In this paper we associate manually the states with the schemes taken into 
consideration the required QoS. For example, the real time nature of "Life Threatening" state makes us to rank the Decision Factors in scheme Alert as: Timeless $>$ Availability $=$ Throughput $>$ Reliability $>$ Security $>$ Cost. In our future research, we intend to introduce a model for automatic such association (mapping). Following, we describe the connectivity schemes:

- Non emergency: It represents the regular transmission of user's monitored vital signs, as data-transfer. It is required an assured transfer of a small amount of data. For that reason, we consider Availability and Reliability as Decision Factors of great importance and Throughput and Timeless as Decision Factors of minor importance. In non-emergency case, Cost is a Decision Factor of strong influence on final decision.

- Notification: "Moderate" state triggers that connectivity scheme, where it is transmitted a greater amount of data. The Decision Factor of Throughput is weighted as well as that of Timeless. This QoS of data transfer could be achieved through multiple ANs, multi-homed connectivity. Different service's flows (vital signs-medical records one) could be divided into discrete traffic flows and each one could be mapped into a different ANs from the selected ones. In that way service achieves comprehensively the required QoS.

- $\quad$ Alert: "Severe" service state requires data transmission as a near real-time service. For that reason we set Timeless as the most important Decision Factor. The greater amount of data that is to be transferred (vital signs, crucial medical records and treatment data plan) make as to place Availability and Throughput as Decision Factors of high importance. "Alert" is one of two emergency situations, where Cost, Security and Reliability are consider of minor importance as Decision Factors. Moreover, the emergency with combination with high requirements of "Severe" service state request for extension of user's connectivity. Hence, Rule-1 introduces into available ANs for selection, the neighboring ANs. Moreover Rule2 permits the selection of a group of best ANs.

- Alarm: In "Life-Threatening" service state, user's vital signs, crucial medical records and treatment data plan should be simultaneously transmitted, in real-time. For that reason Decision Factors of Timeless and Throughput influence decisively the selection of best ANs. The emergency of service state is such that Decision Factors of Availability and Reliability are considered of middle importance. The final decision is not affected by Decision Factors of Cost and Security. The implementation of Rules 1 and 2 declare that the whole set of discovered ANs are available for selection and there are no restrictions regarding the number and type of selected.

An experimental proof of our claim as well as a complete demonstration of ABC's incorporated in pHealth aspect is planned for the near future.

\section{Conclusion}

The implementation of the forthcoming pHealth services is a multidisciplinary task that integrates healthcare, communication and computing technologies. This work exploits them in order to establish actual personalized healthcare services. 


\section{References}

1. Hassan, W.H., Hassan, N., Fisal, A.: Tracking Mechanism Using Agents For Personal Mobility in IP Networks. In: 6th International Conference on Industrial Informatics, pp. 995-999 (2008)

2. Song, Q., Jamalipour, A.: A network selection mechanism for Next Generation Networks. In: IEEE International Conference of Communication, vol. 2, pp. 1418-1422 (2005)

3. Sun, J.-Z., Sauvola, J.: From Mobility Management to Connectivity Management. In: Proc. 10th IEEE Symposium on Computers and Communication, pp. 307-312 (2005)

4. Satty, T.L.: The analytic hierarchy process. McGraw-Hill Book Co., New York (1980)

5. Jesus, V., Sargento, S., Aguiar, R.L.: Any-Constraint Personalized Network Selection. In: IEEE 19th International Conference of Personal, Indoor and Mobile Radio Communications (2006)

6. Inoue, M., Wu, G., Mahmud, K., Murakami, H., Hasegawa, M.: Development of MIRAI system for heterogeneous wireless networks. In: 13th IEEE International Symposium on Personal, Indoor and Mobile Radio Communication, vol. 1, pp. 69-73 (2002)

7. Fengou, M.-A., Mantas, G., Lymberopoulos, D., Komninos, N.: Ubiquitous Healthcare Profile Management Applying Smart Card Technology. In: Nikita, K.S., Lin, J.C., Fotiadis, D.I., Arredondo Waldmeyer, M.-T. (eds.) MobiHealth 2011. LNICST, vol. 83, pp. 248-255. Springer, Heidelberg (2012) 